\title{
Qualidade de vida no trabalho dos profissionais de enfermagem em unidade de internação pediátrica
}

\author{
Quality of life in the work of nursing professionals in a \\ pediatric intervention unit
}

\author{
Roseane Pereira Jesus Lima ${ }^{1}$, Ariana Nunes Silva ${ }^{2}$, Augusto César Costa Cardoso ${ }^{3}$ \\ ${ }^{1}$ Autora para correspondência. Escola Bahiana de Medicina e Saúde Pública, Salvador, Bahia, Brasil. ORCID: 0000-0002-0725-5140. roseanepjl@gmail.com \\ 2Escola Bahiana de Medicina e Saúde Pública, Salvador, Bahia, Brasil. ORCID: 0000-0001-8041-5975. nunesare@yahoo.com.br \\ ${ }^{3}$ Escola Bahiana de Medicina e Saúde Pública, Salvador, Bahia, Brasil. ORCID: 0000-0002-5245-7833. acccardoso@uneb.br
}

\begin{abstract}
RESUMO | OBJETIVO: Esta pesquisa tem por objetivo analisar a qualidade de vida no trabalho dos profissionais de Enfermagem da pediatria, considerando os domínios: físicos/ saúde, psicológico, pessoal e profissional. MÉTODO: Estudo transversal descritivo de abordagem quantitativa, com amostra por conveniência, desenvolvido no período de março a maio de 2018, utilizando duas categorias Enfermeiros e Técnicos de Enfermagem da pediatria em unidade de internação hospitalar, com coleta de dados mediante o instrumento Quality of Working Life Questionnaire, conforme a estratégia de snowball sampling ("Bola de Neve"). RESULTADOS: Foram encontrados oito profissionais de cada categoria, com faixa etária média entre 28-32 anos e renda salarial entre dois e três salários mínimos. Quanto a qualidade de vida no trabalho verificou-se o dominio profissional com escore médio de 42,38 classificando como insatisfatório, no dominio fisico/saúde, psicologico e qualidade de vida no trabalho total observou-se escore de neutralidade entre os profissionais avaliados, enquanto que o dominio pessoal obteve um escore satisfatorio. CONCLUSÃO: Os fatores como remuneração, carga horaria excessiva, jornada de trabalho, sono e suas necessidades fisiológicas afetadas interferem diretamente na qualidade de vida no trabalho desses profissionais.
\end{abstract}

DESCRITORES: Qualidade de vida. Trabalho. Pediatria. Satisfação no trabalho. Gestão hospitalar.

\begin{abstract}
OBJECTIVE: This study aims to analyze the quality of life in the work of pediatric nursing professionals, considering the domains: physical/health, psychological, personal and professional. METHOD: Descriptive cross-sectional study of a quantitative approach, with convenience sample, developed from March to May 2018, using two categories of Nursing and Nursing Technicians of the pediatrics in the hospital admission unit, with data collection using the Quality of Working Life Questionnaire according to the strategy of snowball sampling ("Snowball"). RESULTS: Eight professionals from each category were found, with a mean age range of 28-32 years and wage income between two and three minimum wages. Regarding the quality of life at work, the professional domain with a mean score of 42.38 was classified as unsatisfactory, in the physical/health, psychological domain and quality of life in the total work, a score of neutrality was observed among the evaluated professionals, while that the personal domain obtained a satisfactory score. CONCLUSION: Factors such as compensation, excessive hours, work hours, sleep and their physiological needs affect directly the quality of life in the work of these professionals.
\end{abstract}

DESCRIPTORS: Quality of life. Work. Pediatrics. Job satisfaction. Hospital administration. 


\section{Introdução}

Segundo a Organização Mundial da Saúde (OMS) a Qualidade de Vida (QV) está relacionada a percepção do indivíduo à sua posição na vida, no contexto da cultura e sistema de valores nos quais ele vive e com relação aos seus objetivos, expectativas, padrões e preocupações, sendo não somente associada ao campo da saúde à ausência da doença, mas também ao bemestar físico, psicológico e social de cada indivíduo'.

A QV pode ser atingida pela busca da satisfação e realização profissional, adquirida por meio do trabalho, já que através dele o indivíduo sente-se importante e produtivo. Porém, quando o mesmo não é executado e organizado de modo satisfatório pode ser causador de fatores desgastantes, estressores que potencializam o adoecimento do trabalhador ${ }^{1,2}$.

A qualidade de vida no trabalho (QVT) está diretamente relacionada à satisfação e ao bem-estar do indivíduo na execução de suas tarefas sendo indispensável no que diz respeito à sua produtividade ${ }^{3}$. Em virtude das demandas físicas, emocionais e mentais decorrentes do trabalho, os profissionais de enfermagem da pediatria estão expostos ao comprometimento de sua qualidade de vida e capacidade laboral ${ }^{3}$.

O Processo de trabalho na pediatria coloca os profissionais de enfermagem frente a diversos fatores que podem afetar sua QVT, dentre os quais se destacam o desgaste emocional, provocados pela vivência de perdas, dores, sofrimento e morte ao cuidarem de crianças e seus familiares, através de condições de trabalho frequentemente insalubres, com escassez de recursos de materiais, ou por subdimensionamento de pessoal na prestação de uma assistência qualificada, além de jornada de trabalho extenuante, falta de reconhecimento e desvalorização profissional.

A intensidade da vivência do trabalho da enfermagem nas unidades de internação pediátricas da rede hospitalar exige do profissional uma contínua e profunda mobilização de energia, domínio das próprias emoções e conhecimento dos limites que, por vários motivos, pode não estar disponível ou pode não ser suficiente para evitar o estresse do profissional. O cuidar exige do profissional de enfermagem, conhecimento técnico científico, habilidades, qualificação e dedicação ao próximo, pois assim proporcionará um ambiente de trabalho satisfatório ${ }^{4}$.
Faz-se fundamental avaliar o indivíduo de maneira biopsicossocial, observando fatores que interferem nesse cotidiano e comprometem suas habilidades desenvolvidas no ambiente de trabalho ${ }^{2}$, considerando-se, portanto, os domínios fisicos/saúde, psicológico, pessoal e profissional, pois, para além dos significados do trabalho, os colaboradores também são influenciados por sua história de vida pessoal e outras subjetividades ${ }^{5}$.

Este estudo tem por objetivo analisar a qualidade de vida no trabalho dos profissionais de enfermagem pediátricos de unidade de internação da rede hospitalar. Será considerado os domínios físico/saúde, psicológico, pessoal e pofissional, justificando-se por três vertentes: a relevância para a sociedade, a relevância para a comunidade acadêmica e a aproximação das autoras com o tema de estudo e como perspectiva contribuir com a produção do conhecimento relativo à QVT e especialmente em relação às profissionais da área pediátrica, haja vista que existe uma lacuna na literatura acerca dessa temática.

\section{Método}

Estudo transversal descritivo de abordagem quantitativa, com amostra por conveniência, desenvolvido no período de março a maio de 2018 , entre enfermeiros e técnicos de enfermagem pediátricos economicamente ativos, maiores de 18 anos que atuam ha mais de um ano em unidades de internação hospitalar em pediatria, sendo excluidos participantes que não tenham completados os questionários e que estejam afastados de suas funções laborais. Foram enviados 65 email-s, respondidos 23, porém, 7 foram excluídos não respeitaram os critérios de inclusão, totalizando uma amostra com 16 profissionais de enfermagem.

A forma de recrutamento para compor a amostra se deu de acordo com a rede de contatos das pesquisadoras, através de um convite aos trabalhadores fora do seu ambiente de trabalho, que por sua vez indicam novos participantes e assim sucessivamente, permitindo o agrupamento dos voluntários da pesquisa a partir de seu contato social através da estratégia de snowball sampling ("Bola de Neve"). 
Uma vez identificado o participante através da rede pessoal, esses trabalhadores foram convidados a responder um questionário de pesquisa por via eletrônica (email), onde constava uma carta-convite com orientaçoes e esclarecimentos do estudo estabelecidos no TCLE. O questionário eletrônico construído foi disponibilizado pela Internet através de um link gerado automaticamente por uma ferramenta, a qual consiste em um software denominado SurveyMonkey -- da plataforma de questionários da conta "Bahiana-Pesquisas" -- que foi enviado para o público-alvo. Em seguida, após o preenchimento da pesquisa pelos participantes, as respostas apareceram imediatamente na plataforma desta ferramenta, sendo exportadas em formato de gráficos e tabela do Microsoft Office Excel.

Foram utilizados os questionários de dados sociodemográficos e qualidade de vida no trabalho. 0 primeiro questionário se refere às informações dos indicadores socioeconômicos, demográficos e ocupacionais, contemplando as orientações do Instituto Brasileiro de Geografia e Estatística (IBGE) e Critério Brasil/ABEP. O segundo consiste na coleta das informações sobre qualidade vida no trabalho, através do Quality of Working Life Questionnaire (QWLQ-bref) idealizado pelos pesquisadores ${ }^{7,8}$, composto por 20 (vinte) perguntas com 5 (cinco) alternativas de respostas classificadas através de uma escala Likert onde 1 equivale a "muito pouco/nada" e 5, a "muito boa/ extremamente". As respostas devem ser consideradas a partir das vivências das duas últimas semanas. Em havendo incerteza da resposta existe a instrução para que se estabeleça a primeira opção que surgiu em mente do respondente.

As perguntas referentes ao QWLQ-bref são de caráter direto e objetivo, classificadas em quatro domínios que compreendem: físico/saúde referente à saúde, doença no ambiente de trabalho e hábitos dos trabalhadores; psicológico refente à satisfação pessoal, autoestima do trabalhador e motivação no trabalho; profissional referente às questões organizacionais que podem influenciar a qualidade de vida dostrabalhadores; pessoal ligado à família, crenças pessoais e religiosas e à cultura e como estes podem influenciar o trabalho.
A análise dos dados quantitativos foi definida pelo instrumento QWLQ-bref, em que índices abaixo de 22,5 são classificados como muito insatisfatório; de 22,5 a 45 como insatisfatórios; de 45 a 55 como neutros; de 55 a 77,5 equivale a satisfatório e acima de 77.5 como muito satisfatório.

Trata-se de um recorte de um projeto maior do grupo de pesquisa GESTRA/GEPIS da Escola Bahiana de Medicina e Saúde Pública, que trata do seguinte tema: Interfaces entre Qualidade de Vida, Saúde do Trabalhador e Organização do processo de trabalho, que foi submetido e aprovado pelo CEP da EBMSP com parecer 2.354.329 de 27 de Outubro de 2017, CAAE: 66111417.0 .0000 .5544 , cumprindo os requisitos da Resolução 466/12 no Conselho Nacional de Saúde.

\section{Resultados}

Para caracterizar a amostra buscou-se evidenciar os aspectos sociodemográficos, econômicos e ocupacionais, conforme a Tabela 1, observando-se que a maioria dos profissionais do sexo feminino, com idade média $28(+/-8)$ anos, que recebe de 2 a 3 salários mínimos (43,75\%) e tem em média de 5 a 10 anos de exercício profissional $(68,7 \%)$, havendo destaque para os profissionais com especialização, dentre os participantes avaliados. Entre os avaliados obtivemos $50,0 \%$ com ensino médio, $12,5 \%$ com superior completo (sem especialização) e 37,5\% ensino superior com especialização. Dentre os pesquisados, 37,5\% são casadas e a maioria tem em média 2 a 3 filhos (60\%), além de metade apresentarem vínculo empregatício celetista e trabalharem com carga horaria em média de 44 horas semanais. 


\begin{tabular}{|c|c|c|c|}
\hline VARIÁVEIS & $\mathrm{N}=16(\%)$ & VARIÁVEIS & $\mathrm{N}=16(\%)$ \\
\hline IDADE & $28(+/-8)$ & PROFISSÃO & \\
\hline $\mathrm{N}^{\circ} \mathrm{FILHOS}$ & 2 & Técnico de enfermagem & $8(50,0)$ \\
\hline SEXO & & Enfermeira & $8(50,0)$ \\
\hline Feminino & $14(87,5)$ & MOD. VÍCULO EMPREGATÍCIO & \\
\hline \multirow[t]{3}{*}{ Masculino } & $2(12,5)$ & & \\
\hline & & Celetista & $8(50,0)$ \\
\hline & & Servidor público & $6(37,5)$ \\
\hline ESTADO CIVIL & & Contratos temporários & $2(12,5)$ \\
\hline Solteira & $4(25,0)$ & RENDA & \\
\hline Casada & $6(37,5)$ & & \\
\hline Divorciada & $1(6,2)$ & 1 a 2 & $3(18,7)$ \\
\hline União estável & $3(18,7)$ & 2 a 3 & $7(43,7)$ \\
\hline Viúvo & $2(12,5)$ & 3 a 4 & $6(37,5)$ \\
\hline ESCOLARIDADE & & CARGA HORÁRIA & \\
\hline Ensino médio completo & $8(50,0)$ & & \\
\hline Ensino superior & $2(12,5)$ & 36 & $8(50,0)$ \\
\hline \multirow[t]{4}{*}{ Ensino superior com especialização } & $6(37,5)$ & 44 & $8(50,0)$ \\
\hline & & TEMPO DE SERVIÇO & \\
\hline & & 2 a 5 ANOS & $6(37,5)$ \\
\hline & & 5 a 10 ANOS & $10(62,5)$ \\
\hline
\end{tabular}

Fonte: Os autores (2018).

Na representação gráfica, em uma escala centesimal, as médias de cada domínio e o índice da QVT global dos Enfermeiros e Técnicos de Enfermagem na pediatria da rede hospitalar foram: No domínio físico/saúde, psiocológico e QVT/total, respectivamente, apresentaram médias com escores de $(51,17 ; 54,17$ e 53,11) e após observar a escala de classficação criada pelo pesquisador ${ }^{7}$, identicou-se que os domínios descritos revelam-se dentro de uma faixa de neutralidade. Observou-se ainda, que o domínio pessoal teve índice de $(64,45)$ classificando-o como satisfatório. O domínio profissional apresenta-se como o mais baixo com escore médio de $(42,63)$, sugerindo a insatisfação dos profissionais da pediatria no âmbito laboral.

Gráfico 1. Médias dos escores de satisfação nos Domínios da QVT dos profissionais de enfermagem da pediatria ( $\mathrm{n=16}$ ), Salvador - Ba, 2018

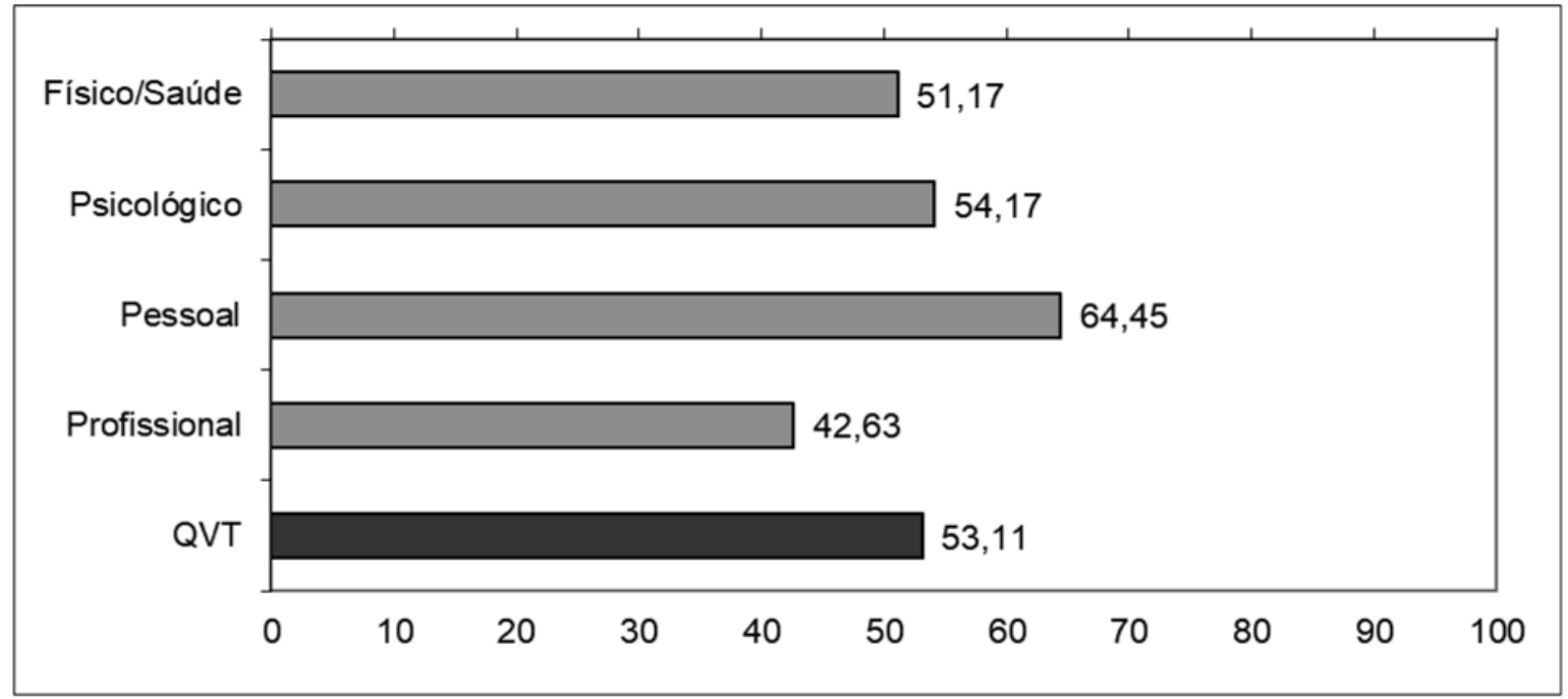

Fonte: Os autores (2018). 


\section{Discussão}

A caracterização dos trabalhadores de enfermagem em unidades de internação pediátrica, envolvidos no estudo confirmam os achados em outras pesquisas, nos quais o maior número de profissionais é do sexo feminino, o que, segundo a literatura seria justificável pelas características históricas, em que o cuidado sempre esteve ligado às mulheres, além das características próprias da maternidade e ao gosto por cuidar de crianças, demostrando maior atuação deste sexo em unidades pediátrica hospitalar ${ }^{9}$.

A idade média dos participantes assim como o estado civil também entraram em conformidade com outros estudos, como os resultados apresentados por Talhaferro ${ }^{10}$, em que os participantes se apresentaram em sua maioria com faixa etária entre $28 \mathrm{e}$ 32 anos e casados (37,5\%). Outro fator foi quanto ao tempo de serviço que em outro estudo mostra um resultado semelhante, onde os profissionais apresentam tempo de atuação entre 2 a 11 anos de serviço, enquanto nossa amostra apresentou tempo de atuação na instituição de 5 a 10 anos $^{10}$.

A carga horária de 44 horas semanais predominou entre os trabalhadores celetistas e de 36 horas semanais entre os servidores público. Isto evidência a existência de condições diferenciadas de trabalho entre os trabalhadores e essa situação também reforça a constatação da desigualdade das relações estabelecidas no trabalho (divisão social do trabalho), mesmo em se tratando do serviço público de saúde. Essas desigualdades nos setores públicos e privados assim como carga horaria extensiva, desligamento automático do serviço, constituem o conjunto de ações caracterizadas como condições precárias de trabalho ${ }^{11,12}$.

Estudos têm ressaltado a importância de fatores diretamente ligados à QV do trabalhador, como: baixo salário, diferentes modalidades de vínculo empregatício acarretando condições laborais precárias, desvalorização profissional, multiplicidade de empregos, dentre outras variáveis, conforme evidenciado em uma revisão bibliográfica realizada nos anos de 1999 a $2012^{13,14}$.

O presente estudo sugere que a percepção da qualidade de vida no trabalho está diretamente relacionada ao bem-estar e a segurança dos indivíduos no ambiente de trabalho sendo assim fundamental para garantir uma maior produtividade e qualidade no trabalho, além de uma maior satisfação na vida pessoal ${ }^{12}$.
É notório que a QVT sofre diversas influências de variáveis, como relações interpessoais no ambiente laboral, satisfação com a remuneração, reconhecimento e valorização da atividade exercida, além da recompensa da concretização dos objetivos alcançados. Estes fatores também contribuem com o comprometimento com o trabalho, a produtividade e a motivação. Da mesma forma, fatores externos ao ambiente de trabaIho como vida pessoal, saúde, lazer e estado emocional influenciam diretamente na QVT13,4,12.

Nesta mesma linha de pensamento pode-se dizer que existe a necessidade de visualizar o ser humano integralmente, e não somente como profissional ou como ser social. A investigação da QVT possui muitos pontos de intersecção com os critérios utilizados para análise da QV de uma forma ampla. Isso implica que a análise da QVT não deve se restringir aos fatores do ambiente laborativo ${ }^{14,15}$.

A amostra desse estudo apresentou-se com uma percepção insatisfatória na QVT no domínio profissional. Para Reis-Jr7, o resultado de menor satisfação no domínio profissional deve considerar que nesse domínio existem fatores relacionados as condições do trabalho, da concessão de benefícios pela empresa. Considerando que neste domínio avaliam-se aspectos relativos a orgulho pela empresa, parceria entre os membros da equipe, autonomia; burocracia; carga horária; cooperação entre níveis hierárquicos; credibilidade do superior; criatividade; educação; equidade interna e externa; estabilidade de horários; habilidade e disponibilidade de empregados; identidade com a tarefa; acidentes de trabalho; informações sobre os processos totais do trabalho; metas e objetivos; nível de desafio, pode-se dizer que essas variáveis são indicadores que impactam na QVT e estão envolvidos com a desmotivação do profissional16,17,20.

Em contrapartida avaliando o domínio pessoal observou-se o índice com escore satisfatório de QVT, demostrando que os profissionais se encontram realizados com o desenvolvimento de suas atividades laborais e as empresas estão proporcionando e impulsionando o funcionário a se auto avaliarem de forma que apresenta uma capacidade para meIhorar sua QVT, corroborando com outras pesquisas, que partem do pressuposto de que as realizações e satisfação pessoal estão intimamente ligadas com a determinação de cada indivíduo ${ }^{18,19}$. 
Para os domínios físicos/saúde, psicológico e QVT total foi encontrado índices evidenciando equilíbrio e ou neutralidade entre o público avaliado. Autores explicam que para o domínio físico/saúde há uma correlação entre a qualidade do sono, necessidades fisiológicas, conforto e estresse ${ }^{18,19}$. O indivíduo para ter uma boa qualidade de vida necessita, no mínimo, de um boa noite de sono e suas NHB garantidas ${ }^{19}$. Já os achados do domino psicológico guardam relação com fatores como: responsabilidade na instituição, orgulho do trabalho, liberdade de expressão, valores e crenças, autocontrole. Autores observam que a partir do momento que o colaborador tem esses fatores ignorados passa a não ter o mesmo desempenho de antes, tornando-se um funcionário triste e desmotivado ${ }^{19,20}$.

A QVT global demostra que o colaborador também se encontra dentro de uma margem de neutralidade tendendo a ascensão para a satisfação. Autores trazem que esses fatores estão intimamente ligados com a satisfação e realização no seu ambiente laboral e suas atividades desenvolvidas, atrelados aos laços familiares e ao bem-estar pessoal ${ }^{21,22}$.

Em geral, uma boa QVT só irá existir se o indivíduo atentar para o seu comportamento em relação a sua saúde e QV como um todo, procurando eliminar ou reduzir os hábitos negativos que comprometem seu bem-estar. Para isso há a necessidade de as instituições terem uma visão geral e mais humanizada em relação ao trabalhador, promovendo um ambiente mais harmonioso e saudável que colaborem para desenvolver uma boa QVT, motivando esse trabalhador a melhor desenvolver suas atividades laborais 20,23,24,25.

Um bom lugar para se trabalhar possibilita, entre outras coisas, que as pessoas tenham, além do trabalho, outros compromissos em suas vidas, como a família, os amigos e os hobbies pessoais ${ }^{25}$. O trabaIhador da equipe de enfermagem pediátrica possui a gratificação em cuidar de crianças, o compromisso de oferecer afeto, carinho e trabalho humanizado. Dessa forma, o profissional canaliza suas experiências, decepções, sentimentos e emoções, ou seja, o estresse do trabalho que ocorre diariamente na assistência à criança hospitalizada, e essa desmotivação impacta na assistência prestada à criança hospitalizada ${ }^{10}$.

\section{Considerações finais}

Após analisar os domínios observou-se que, carga horária excessiva de trabalho, múltiplos vínculos, qualidade no sono, além da falta de reconhecimento profissional, insatisfação no ambiente laboral, foram identificados como indicadores que interferem diretamente na QVT, destacando-se assim a necessidade, tanto para os trabalhadores quanto para as gestões de diversas organizações, as quais devem redefinir estratégias que possam impactar diretamente na prevenção e promoção à saúde do trabalhador.

Os dominios dentro da faixa de neutralidade denotam o quão os colaboradores estão desmotivados com as atividades desenvolvidas no ambiente laboral. No que se refere à pediatria enquanto uma especialidade da Enfermagem isto deve ser visto com muito cuidado e de maneira minuciosa, levando-se em consideração suas particularidades e, principalmente, o intenso desafio para os profissionais que desenvolvem suas tarefas em hospitais, respeitando os principios éticos, o cuidado das crianças no momento de fragilidade causado pelo internamento, além da pressão dos familiares e cuidadores que os acompanham.

O adoecimento dos trabalhadores de enfermagem pediátricos de unidade de internação da rede hospitalar pode estar associado a diversos fatores, dentre os quais os baixos salários associados com a opção por mais de um emprego, o que determina a permanência no ambiente dos serviços de saúde na maior parte do tempo de suas vidas produtivas. Essa situação pode levar ao aumento do período de exposição aos riscos existentes nesses locais, ampliando o prejuízo para sua QVT, além de diminuição de tempo para atividades de lazer e recreação, necessárias para a manutenção da saúde física e mental.

Os resultados do estudo não podem ser generalizados, mas acredita-se na possibilidade de contribuírem com o conhecimento dos profissionais para a melhoria na qualidade de vida laboral de Enfermeiros e Técnicos de Enfermagem na pediatria de unidades hospitalares. 


\section{Contribuição dos autores}

Lima RPJ participou da concepção, delineamento, pesquisa bibliográfica, construção das Tabelas, interpretação dos resultados, análise estatística dos resultados da pesquisa, além da redação e submissão do artigo científico. Silva AN participou da concepção, delineamento, , pesquisa bibliográfica, , construção das Tabelas, busca e análise estatística dos resultados da pesquisa, além da interpretação dos resultados e da redação do artigo científico. Cardoso ACC participou da orientação e revisão do projeto de pesquisa, da construção do instrumento de investigação, da aprovação da pesquisa no CEP, da interpretação dos resultados, da orientação e da redação do artigo para a submissão e das correções do artigo após a submissão.

\section{Conflitos de interesses}

Nenhum conflito financeiro, legal ou político envolvendo terceiros (governo, empresas e fundações privadas, etc.) foi declarado para nenhum aspecto do trabalho submetido (incluindo mas não limitando-se a subvenções e financiamentos, participação em conselho consultivo, desenho de estudo, preparação de manuscrito, análise estatística, etc.).

\section{Referências}

1. Manzieri DS, Andrade LF et al. Estudo dos efeitos do Lian Gong em 18 Terapias na qualidade de vida de trabalhadores de uma empresa siderúrgica do Município de São Paulo. 78a.ed. São Paulo: Revista Fisiobrasil; 2006.

2. Costa AM, Duarte E. Atividade física e a relação com a qualidade de vida de pessoas com seqüelas de Acidente Vascular Cerebral Isquêmico (AVCI). Rev Bras Ciên Mov. 2002;10(1):47-54. doi: $\underline{10.18511 / \mathrm{rbcm} . \mathrm{v} 10 \mathrm{i} 1.415}$

3. Sampaio JR. Qualidade de Vida no Trabalho: Perspectivas e Desafios Atuais. Revista Psicologia:: Organizações e Trabalho. 2012;12(1):121-136.

4. Sampaio JR. Qualidade de vida no trabalho e psicologia social. São Paulo: Casa do Psicólogo; 2004.

5. Cortez HBT. Qualidade de vida no trabalho sob a ótica da macroergonomia [dissertação]. Porto Alegre: Universidade Federal do Rio Grande do Sul; 2004.

6. Brasil. Conselho Federal de Enfermagem. Projeto Lei 2295/2000. Dispõe sobre a jornada de trabalho dos Enfermeiros, Técnicos e Auxiliares de Enfermagem. Brasília, DF: Ministério da Saúde; 2004.

7. Reis Junior DR. Qualidade de vida no trabalho: construção e validação do questionário QWLQ-78. [dissertação]. Ponta Grossa:Programa de Pós-Graduação em Engenharia de Produção,

Universidade Tecnológica Federal do Paraná; 2008.
8. Cheremeta M, Pedroso B, Pilatti LA, Kovaleski JL. Construção da versão abreviada do QWLQ-78: um instrumento de avaliação da qualidade de vida no trabalho. Revista Brasileira de Qualidade de Vida. 2011;3(1):1-15. doi: 10.3895/S2175-08582011000100001

9. Oler FG, Vieira MRR. O conhecimento da equipe de enfermagem sobre a criança hospitalizada. Arq Ciênc Saúde. 2006;13(4):192-197.

10. Caldas CB, Somensari P, Costa SN, Siqueira MMM, Claro JACS. Satisfação e engajamento no trabalho: docentes temáticos e auxiliares da EAD de universidade privada brasileira. Revista Interinstitucional de Psicologia. 2013;6(2):225-237.

11. Luedy A, Mendes VLPS, Ribeiro Júnior H. Gestão pública por resultados: contrato de gestão como indutor de melhorias em um hospital universitário. Organ Soc. 2012;19(63):641-659. doi: 10.1590/S1984-92302012000400005

12. Timossi LS, Francisco AC, Santos Junior G, Xavier AAP. Análise de qualidade de vida no tabalho de colaboradores com diferentes níveis de instrução através de uma análise de correlações. Prod. 2010; 20(3):471-480. doi: 10.1590/S0103-65132010005000031

13. Souza MA, Stancato K. Avaliação da qualidade de vida de profissionais de saúde em Campinas. Rev Adm Saúde. 2010;12(49):154-162.

14. Cavassani AP, Cavassini EB, Biazin CC. Qualidade de vida no trabalho: fatores que influenciam as organizações. In: Anais eletrônicos do XIII SIMPEP - Simpósio de Engenharia de Produção, 2006; Bauru: UNESP; 2006.

15. Brasil. Ministério do trabalho e Emprego. Norma regulamentadora NR 32, de 11 de novembro de 2005. Dispõe sobre a segurança e saúde no trabalho em Serviço de Saúde. Brasília: Ministério do trabalho e Emprego; 2005.

16. Farias SNP, Zeitoune RCG. A qualidade de vida no trabalho de enfermagem. Esc Anna Nery R Enferm. 2007;11(3):487-93. doi: 10.1590/S1414-81452007000300014

17. Detoni DJ. Estratégias de avaliação da qualidade de vida no trabalho: estudos de casos em agroindústrias [dissertação]. Florianópolis: Universidade Federal de Santa Catarina; 2001.

18. Limongi-França AC. Qualidade de Vida no Trabalho - QVT: conceitos e práticas nas empresas da sociedade pós-industrial. 2.ed. São Paulo: Atlas; 2004.

19. Vieira MJ. Imagem cultural e motivação na escolha da enfermagem. Aracaju: EDUFS/ Fund. Oviêdo Teixeira; 2002. p. $19-50$

20. Nahas MV. Atividade Física, saúde e qualidade de vida: conceitos e sugestões para um estilo de vida ativo. 2.ed. Londrina: Midiograf; 2001. 
21. Limongi-França AC, Zaima G. Gestão de Qualidade de Vida no Trabalho - GQVT. In: Manual de Gestão de pessoas e equipes: estratégias e tendências. São Paulo: Gente; 2002.

22. Machado CLB. Motivação, Qualidade de Vida e participação no trabalho [dissertação]. Florianópolis: Universidade Federal de Santa Catarina; 2002.

23. Gutierrez GL. Por Que é tão difícil participar? O exercício da participação do campo educacional. São Paulo: Paulus; 2004.

24. Rego A, Moreira JM, Felício MJ, Souto SO. As melhores organizações para trabalhar: um domínio especifico da responsabilidade social das empresas. Papeles de ética, economia ydirección. 2003;8:1-5.

25. Levering R. Um excelente lugar para se trabalhar: o que torna alguns empregadores tão bons (e outros tão ruins). Rio de Janeiro: Qualitymark, 1986. 